\title{
NOTE ON $\Gamma^{*}$-SEMIGROUPS
}

\author{
BY TAKAYUKI TAMURA
}

Communicated by Edwin Hewitt, February 20, 1962

The system $L(S)$ of all nonvoid subsemigroups of a semigroup $S$ is generally a semilattice ${ }^{1}$ with respect to the inclusion relation. $L(S)$ is called the subsemigroup semilattice of $S$. In the previous paper [1] we determined all the $\Gamma$-semigroups, ${ }^{2}$ i.e., the semigroups whose subsemigroup semilattices are chains. In detail, all the types of $\Gamma$ semigroups are

(1.1) cyclic groups $G\left(p^{n}\right)$ of order of prime power,

(1.2) quasi-cyclic groups $G\left(p^{\infty}\right)$,

(1.3) unipotent semigroups generated by $d$ with each of the following defining relations:

(1.3.1) $d^{2}=d^{3}$

(1.3.2) $d^{3}=d^{4}$

(1.3.3) $d^{2}=d^{p^{m}+2}, p$ prime,

(1.3.4) $d^{3}=d^{p^{m}+3}, p$ prime $\neq 2$.

In the present note, we shall define $\Gamma^{*}$-semigroups as generalizations of $\Gamma$-semigroups and shall report the structure of $\Gamma^{*}$-semigroups except for a part of infinite $\Gamma^{*}$-groups. The proof will be omitted here but will be given elsewhere. ${ }^{3}$

Definition. A semigroup $S$ is called a $\Gamma^{*}$-semigroup if every subsemigroup different from $S$ is a $\Gamma$-semigroup.

$S$ is a $\Gamma^{*}$-semigroup if and only if $L(S)$ is a semilattice satisfying:

Any subset which contains the greatest element is a subsemilattice. A semilattice of this kind is called a $C_{0}$-semilattice. Obviously all the semigroups of order 2 are $\Gamma^{*}$-semigroups, and a homomorphic image of a $\Gamma^{*}$-semigroup is also a $\Gamma^{*}$-semigroup.

LemMa 1. Every element of a $\Gamma^{*}$-semigroup is of finite order, that is, for any element $x$ there is an idempotent $e$ and a positive integer $n$ such that $x^{n}=e$.

Lemma 2. $A \Gamma^{*}$-semigroup of order $>2$ is unipotent. (i.e., an idempotent element is unique).

Generally a unipotent semigroup any element of which is of finite

\footnotetext{
${ }^{1}$ By a semilattice we mean a partially ordered set in which there is a join of two elements.

2 In [1] we called them $\Gamma$-monoids.

3 Semigroups and their subsemigroups semilattices, to appear.
} 
order is determined by a group and a $Z$-semigroup (i.e., a unipotent semigroup with zero) $[2 ; 3]$. By Lemmas 1 and 2 , we can make the discussion proceed to $\Gamma^{*}$-Z-semigroups, $\Gamma^{*}$-groups, and then to the general cases.

Theorem 1. Any $\Gamma^{*}$-Z-semigroup is of order $\leqq 4$. All the types of $\Gamma^{*}$-Z-semigroups other than $\Gamma$-semigroups are listed as follows:

(2.1) $\{0, a, b\}$ of order 3 where $x y=0$ for all $x, y$,

(2.2) $\{0, a, b, c\}$ of order 4 defined as

(2.2.1) $b^{2}=c^{2}=a$ and other products $=0$.

(2.2.2) $b^{2}=c b=c^{2}=a$ and other products $=0$.

(2.2.3) $b^{2}=c^{2}=b c=c b=a$, and other products $=0$.

As far as the $\Gamma^{*}$-groups are concerned, we shall limit ourselves to the case of $\Gamma^{*}$-groups which are properly homomorphic to $\Gamma$-groups.

We can prove that any $\Gamma^{*}$-group which is properly homomorphic to a $\Gamma$-group has a normal subgroup of index of a prime number. Making use of the theory of finite groups $[4 ; 5 ; 6]$, we have

Theorem 2. Any $\Gamma^{*}$-group, which is not a $\Gamma$-group and is homomorphic to a $\Gamma$-group of order $>1$, has one of the following types.

(3.1) The groups of order $p q$ where $p$ and $q$ are different primes. There are two types (3.1).

(3.2) The elementary abelian group: $G(p) \times G(p)$.

(3.3) The generalized quaternion group of order 8.

Incidentally a finite $\Gamma^{*}$-group, which is not a $\Gamma$-group, is homomorphic to a $\Gamma$-group; a commutative $\Gamma^{*}$-group which is not a $\Gamma$ group is the direct product of two groups of prime order. Consequently we see that the result of Theorem 2 includes the cases where a $\Gamma^{*}$-group is homomorphic to a nontrivial finite group or a commutative group. However the problem of determination of the remaining case is still open.

Next, let $S$ be a unipotent $\Gamma^{*}$-semigroup which is neither a group nor a $Z$-semigroup. Then we can prove that $S$ must be finite. The kernel (i.e., the least ideal) of $S$ is of type $G\left(p^{n}\right)$, and the difference semigroup $D$ of $S$ modulo $G\left(p^{n}\right)$, due to Rees [7] is a $Z$-semigroup which has one of the types (1.3.1), (2.1), (2.2.1), (2.2.2), (2.2.3).

Let $e$ be the unique idempotent of $S$, and let $d$ be a generator of $D$. $G\left(p^{n-1}\right)$ will denote the subgroup of order $p^{n-1}$ of $G\left(p^{n}\right)$.

TheOREM 3. When $G\left(p^{n}\right)$ is given, we can determine all the unipotent $\Gamma^{*}$-semigroups, non $\Gamma$-semigroups, whose kernel is $G\left(p^{n}\right)$, by the product of $e$ and $d$ in the following way. 
(4.1) In the case $D$ of order $2, S=G\left(p^{n}\right) \cup\{d\}, n \neq 0$,

$$
e d \in G\left(p^{n-1}\right)-G\left(p^{n-2}\right) .4
$$

(4.2) In the case $D$ of order $3, D$ is of type (2.1) and $S=G\left(p^{n}\right)$ $\cup\left\{d_{1}, d_{2}\right\}, n \neq 0$.

(4.2.1) $e d_{1}=e d_{2} \in G\left(p^{n}\right)-G\left(p^{n-1}\right)$,

(4.2.2) $p^{n} \neq 2, e d_{1} \neq e d_{2} ; e d_{1}, e d_{2} \in G\left(p^{n}\right)-G\left(p^{n-1}\right)$.

(4.3) In the case $D$ of order $4, S=G\left(p^{n}\right) \cup\left\{d_{1}, d_{2}, d_{3}\right\}, d_{2}^{2}=d_{3}^{2}=d_{1}$, $n \neq 0, p \neq 2$.
(4.3.1) $D$ of $(2.2 .1)$
(4.3.2) $D$ of (2.2.2) $e d_{2}=e d_{3} \in G\left(p^{n}\right)-G\left(p^{n-1}\right)$.
(4.3.3) $D$ of (2.2.3)

According to the above-mentioned theorems, we see that if $S$ is a finite $\Gamma^{*}$-semigroup, the finite $C_{0}$-semilattice $L(S)$ satisfies JordanDedekind condition (or $J$-condition cf. [8]). Generally a finite $C_{0^{-}}$ semilattice $K$ satisfying $J$-condition is called a $C_{0} J$-semilattice. Let $\delta$ denote the dimension of $K$ (cf. [8]), $\lambda$ the breadth, i.e., the number of the maximal chains in $K$, and $\mu$ the order, i.e., the number of elements of $K$.
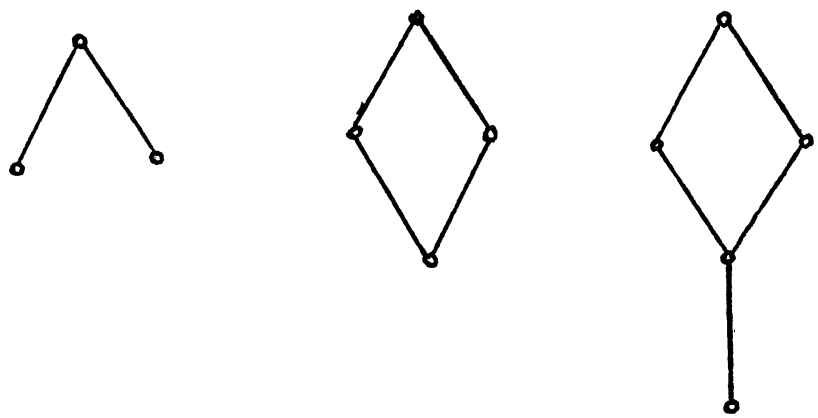

r-Semigroups

Idempotent Semigroups of order 2

(2.1), (3.1)

TheOREM 4. A finite $C_{0} J$-semilattice $K$ is isomorphic to certain $L(S)$ for some finite $\Gamma^{*}$-semigroup $S$ if and only if $\delta, \lambda$, and $\mu$ satisfy the following conditions.

(5.1) $\delta+\lambda-\mu=0$,

(5.2) $\lambda=\alpha+1$ where $\alpha=0$ or 1 or any prime number,

${ }^{4} A-B$ denotes the set of elements of $A$ which are not in $B$. 


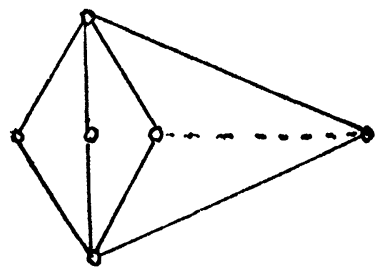

$(3.1),(3.2)$

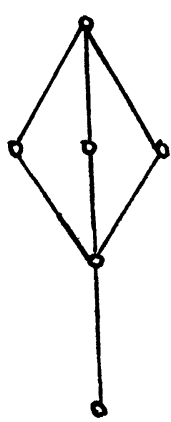

(3.3)

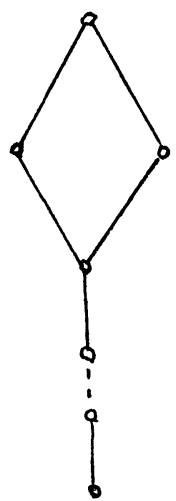

(4.1), (4.2), (4.3)

(5.3) $\left\{\begin{array}{l}\text { if } \lambda=1 \text { or } 2, \text { then } \delta \text { can } b \\ \text { if } \lambda=3, \text { then } \delta=2 \text { or } 3,\end{array}\right.$

if $\lambda=p+1, p$ being a prime number $>2$, then $\delta=2$.

Finally we shall show the diagrams of $L(S)$ for a finite $\Gamma^{*}$-semigroup $S$.

\section{REFERENCES}

1. T. Tamura, On a monoid whose submonoids form a chain, J. Gakugei Coll. Tokushima Univ. 5 (1954), 8-16.

2. - Note on unipotent inversible semigroups, Kōdai Math. Sem. Rep. 3 (1954), 93-95.

3. - The theory of construction of finite semigroups. III, Osaka Math. J. 10 (1958), 191-204.

4. M. Hall, The theory of groups, Macmillan, New York, 1959.

5. H. Zassenhaus, The theory of groups, 2nd ed., Vandenhoeck and Ruprecht, Göttingen, 1956.

6. M. Osima, Group theory, Kyoritsusha, Tokyo, 1954 (Japanese).

7. D. Rees, On semigroups, Proc. Cambridge Philos. Soc. 36 (1940), 387-400.

8. G. Birkhoff, Lattice theory, Amer. Math. Soc. Colloq. Publ., Vol. 25, Amer. Math. Soc., New York, 1948.

TOKUSHIMA UNIVERSITY AND

University of California, Davis 